\title{
TRPS1 mutation associated with trichorhinophalangeal syndrome type 1 with 15 supernumerary teeth, hypoplastic mandibular condyles with slender condylar necks and unique hair morphology
}

\author{
Piranit Nik KANTAPUTRA, ${ }^{1,2}$ (D) Dhirawat JOTIKASTHIRA, ${ }^{3}$ Bruce CARLSON, ${ }^{4}$ \\ Teerapat WONGMANEERUNG,${ }^{5}$ Natalina QUARTO,${ }^{6}$ Theerapong KHANKASIKUM, ${ }^{7}$ \\ Warit POWCHAROEN, ${ }^{8}$ Worrachet INTACHAI, ${ }^{1}$ Kanich TRIPUWABHRUT ${ }^{3}$ \\ ${ }^{1}$ Division of Pediatric Dentistry, Department of Orthodontics and Pediatric Dentistry, Faculty of Dentistry, Chiang Mai University, \\ ${ }^{2}$ Dentaland Clinic, ${ }^{3}$ Division of Orthodontics, Department of Orthodontics and Pediatric Dentistry, Faculty of Dentistry, Chiang Mai \\ University, Chiang Mai, Thailand, ${ }^{4}$ Department of Anatomy and Cell Biology, University of Michigan, Ann Arbor, Michigan, USA, \\ ${ }^{5}$ Dental Department, Pasang Hospital, Lamphun, Thailand, ${ }^{6}$ Department of Surgery, Division of Plastic and Reconstructive Surgery, \\ Stanford University School of Medicine, Stanford, California, USA, ${ }^{7}$ Dental Department of Mae Sod Hospital, Tak, ${ }^{8}$ Department of \\ Oral and Maxillofacial Surgery, Faculty of Dentistry, Chiang Mai University, Chiang Mai, Thailand
}

\begin{abstract}
Trichorhinophalangeal syndrome type 1 (TRPS1; Online Mendelian Inheritance in Man \#190350) is an autosomal dominant disorder caused by mutations in TRPS1. We report a Thai male with TRPS1 who carried a c.1842C>T (p.Arg615Ter) mutation. He had 15 supernumerary teeth, double mental foramina, hypoplastic mandibular condyles with slender condylar necks and unique ultrastructural hair findings. Body hair was absent. The hair in the area of a congenital melanocytic nevus had a greater number of hair cuticles than normal. Occipital hair had abnormal hair follicles and cuticles. The scale edges of the hair cuticles were detached and rolled up. Hypoplastic mandibular condyles with slender condylar necks, double mental foramina and the rolled up edges of hair cuticles have not been reported in patients with TRPS1.
\end{abstract}

Key words: abnormal hair cuticle, abnormal temporomandibular joint, double mental foramina, supernumerary teeth, trichorhinophalangeal syndrome type 1.

\section{INTRODUCTION}

Trichorhinophalangeal syndrome type I (TRPS1; Online Mendelian Inheritance in Man \#190350) is an autosomal dominant disorder caused by mutations in TRPS1. Characteristic features of the patients include sparse and slow-growing scalp hair, bulbous tip of nose, short stature, brachydactyly and cone-shaped epiphyses of the phalanges. ${ }^{1,2}$ Here, we report a patient with a TRPS1 mutation who had 15 supernumerary teeth, double mental foramina, hypoplastic mandibular condyles with slender condylar necks and a unique hair morphology.

\section{CASE REPORT}

A 21-year-old Thai man of normal intelligence presented with malocclusion (Fig. 1a). He was the second child of non-consanguineous healthy parents, with a normal sister. His height was $155 \mathrm{~cm}(<3$ percentile) and disproportionate short stature was noted (Fig. 1b). He was born with no scalp hair or eyebrows (Fig. 1C). Pubic hair and axillary hair first appeared at age 15 years. At age 21 years, he had no body hair including mustache and beard. His scalp hair was fine, sparse and slowgrowing, with alopecia in the occipital area (Fig. 1d). There was a large hairy congenital melanocytic nevus (CMN) on his left thigh (Fig. 1e). This CMN was $2 \mathrm{~cm}$ in diameter at birth, and it became larger with age. Brachydactyly of the hands and feet was noted. Fingernails and toenails were narrow and slowgrowing (Fig. 1f-g). Radiographic examination showed coneshaped epiphyses of the middle phalanges of the fingers, short first metatarsal bones, short middle and distal phalanges of the toes, fusion of the middle and distal phalanges 3,4 and 5 on the right and left toes, and an unremarkable pelvis (Fig. $2 b-d$ ).

Oral examination showed two erupted supernumerary maxillary permanent teeth, severe crowded teeth, and anterior and

Correspondence: Piranit Nik Kantaputra, D.D.S., M.S., Division of Pediatric Dentistry, Department of Orthodontics and Pediatric Dentistry, Faculty of Dentistry, Chiang Mai University, 239 Huay Kaew Road, Muang District, Chiang Mai 50200, Thailand. Email: dentaland17@gmail.com Received 23 January 2020; accepted 29 March 2020. 


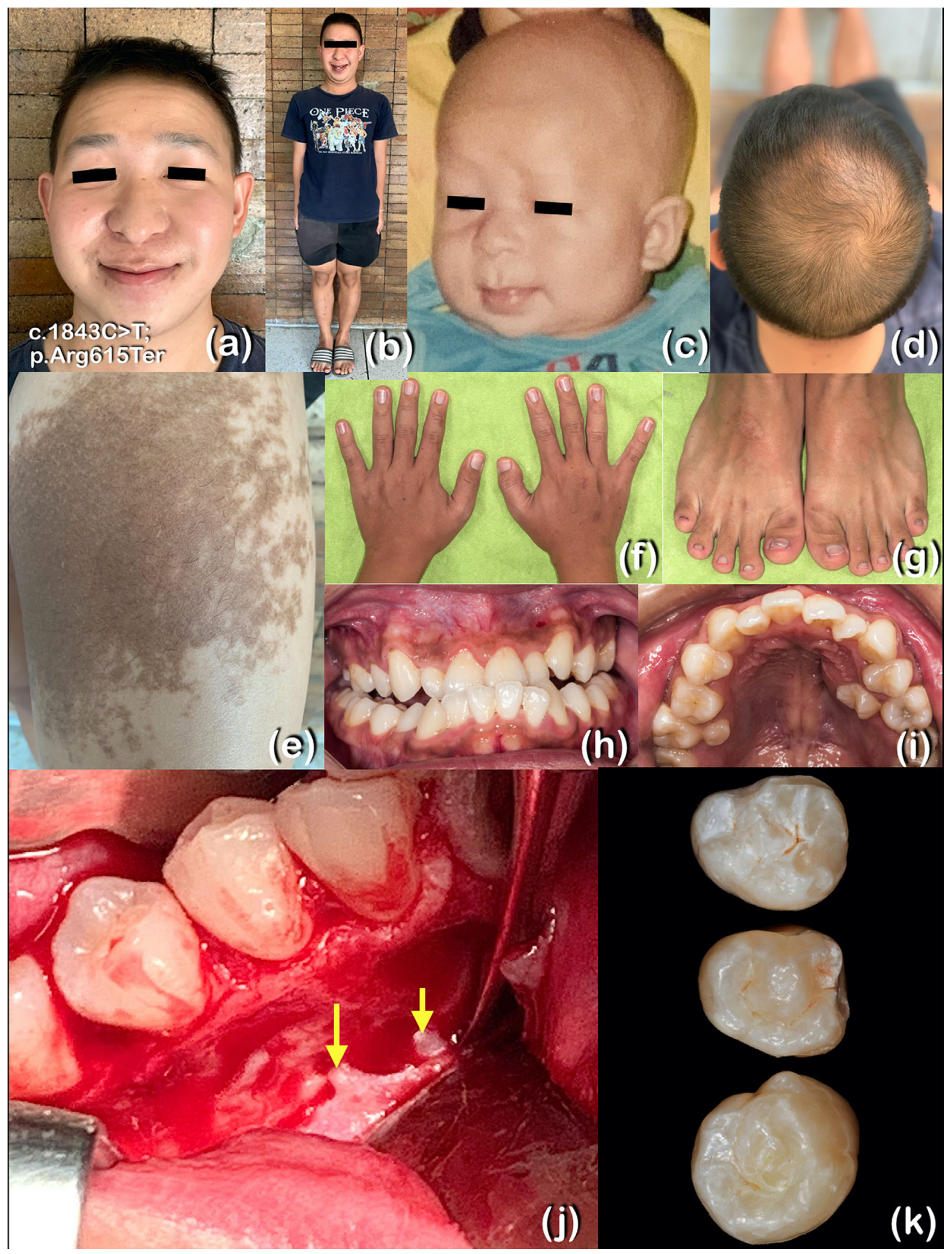

Figure 1. Patient with TRPS I at age 21 years. (a) Bulbous tip of nose and thin vermillion border of upper lip. (b) Disproportionate short stature. The upper part of the body is longer than the lower part. (c) He was born with no scalp hair. (d) His scalp hair is fine, sparse and slow-growing, with alopecia in the occipital area. (e) A large hairy congenital melanocytic nevus on his left thigh. Brachydactyly of f) hands and (g) feet. Fingernails and toenails are narrow and slow-growing. (h) Anterior and posterior open bite. (i) Severe crowding of teeth. Two erupted supernumerary maxillary permanent teeth. (j) Two nerve bundles come out from double mental foramina (arrows). (k) The morphology of all extracted supernumerary teeth resemble that of premolars. 


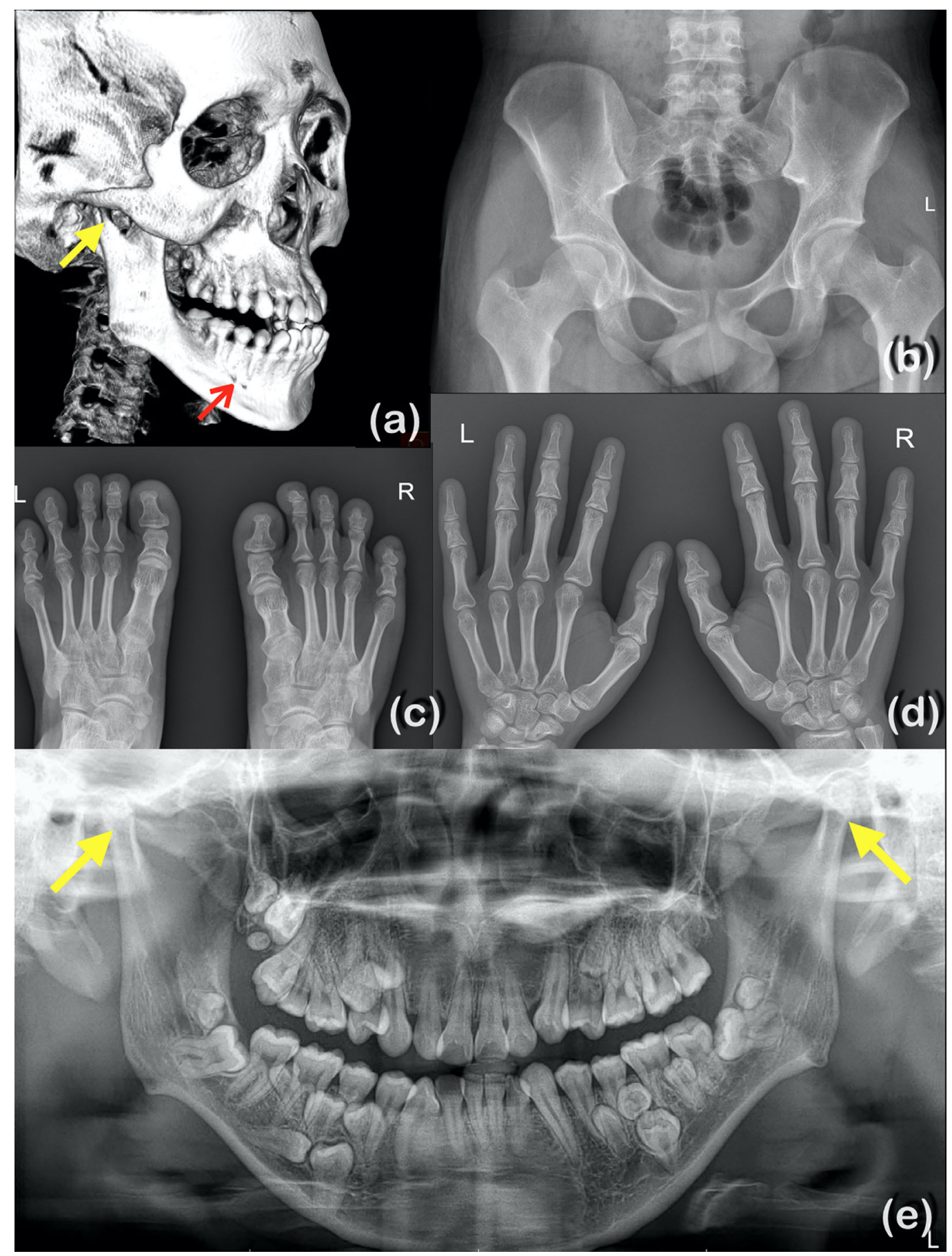

Figure 2. Radiographs of patient at age 21 years. (a) Cone-beam computed tomography shows double mental foramina on the right side (red arrow) and hypoplastic right mandibular condyle with slender condylar neck (yellow arrow). (b) Pelvis is unremarkable. (c) Short first metatarsal bones, short middle and distal phalanges of toes, and fusion of middle and distal phalanges 3, 4, 5 on the right and left toes. (d) Middle phalanges 2-5 are short. Cone-shaped epiphyses of the middle phalanges of fingers. (e) Fifteen supernumerary teeth with premolar morphology. All are located in the premolar and molar areas. Hypoplastic mandibular condyles with slender condylar necks (arrows).

posterior open bite (Fig. 1h,i). Lateral cephalometric analysis revealed short anterior cranial base length, short mandibular length and midface hypoplasia. Cone-beam computed tomography and panoramic radiography showed 15 supernumerary teeth, double mental foramina on the right side and hypoplastic mandibular condyles with slender condylar necks 


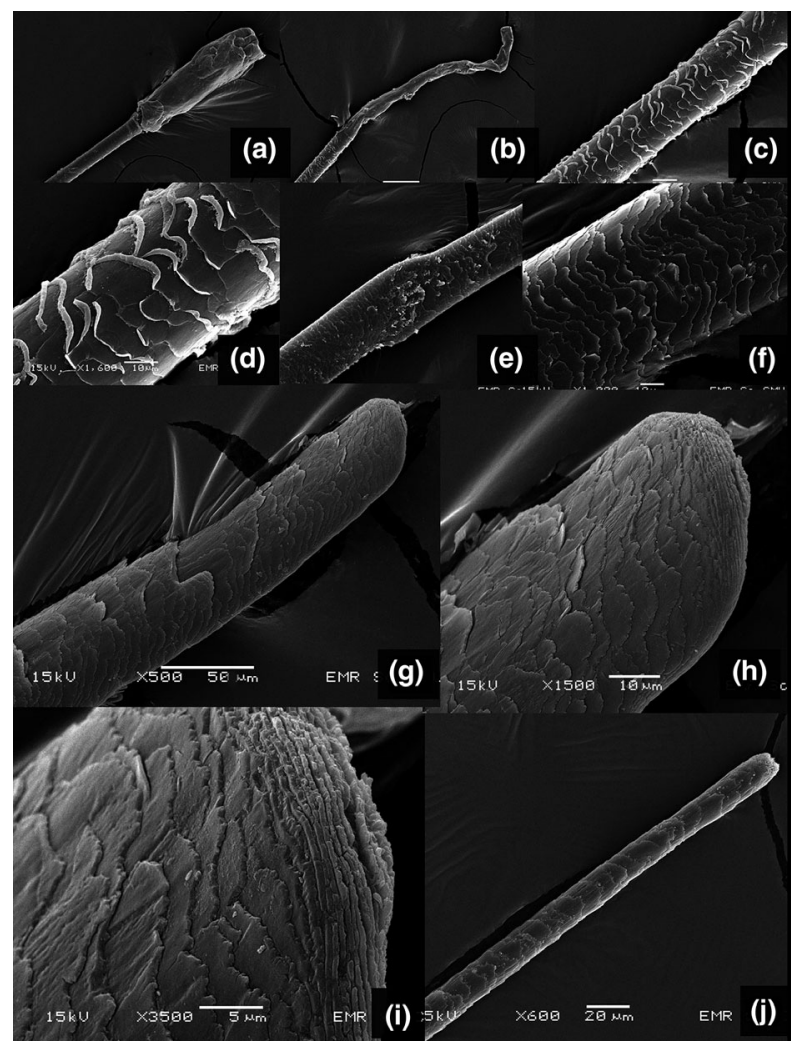

Figure 3. Scanning electron micrographs. (a) Vertex hair. Normal hair follicle with hypoplastic hair cuticles. (b) Occipital hair. Hypoplastic and twisted hair follicles. (c-d) Occipital hair. The scale edges of the hair cuticles detach from the inner root sheath and roll up. (e-f) Hair from the CMN has a greater than normal number of cuticles than those of normal hair. (g-i) Hair from the CMN. The edges of the hair cuticles appearserrated. The tip of the hair is comprised of a stack of hair cuticles. (j) Axillary hair appear normal.

(Fig. 2a,e). The morphology of all extracted supernumerary teeth resembled that of premolars (Fig. 2e,1k). Two nerve bundles came out from double mental foramina (Fig. 1j).

Scanning electron micrographs of his vertex hair showed normal hair follicles and cuticles (Fig. 3a). The occipital hair had hypoplastic and twisted hair follicles. The scale edges of the hair cuticles were detached from the inner root sheath and rolled up (Fig. 3b-d). Hair from the CMN had a greater than normal number of cuticles than those of normal hair. The edges of the hair cuticles appeared serrated (Fig. 3e-i). The tip of the hair was comprised of a stack of hair cuticles (Fig. 3h,i). His axillary hair appeared normal (Fig. 3j).

\section{Mutation analysis}

Mutation analysis of TRPS1 showed a de novo heterozygous base substitution, c.1842C $>\mathrm{T}$ (p.Arg615Ter; rs121908431) (Fig. S1). Another TRPS1 variant (c.2458C >G; p.Pro820Ala; rs78472570) was found in exon 5 (Fig. S2). The allele frequency of this variant according to the Genome Aggregation Database was 0.0008516 . This rare variant was found in his unaffected father and sister. His mother had none of those variants.

\section{DISCUSSION}

We report a man with TRPS1 who carried a de novo heterozygous base substitution c.1842C $>\mathrm{T}$; p.Arg615Ter in TRPS1, which is predicted to cause a stop codon at codon 615 and premature truncation of TRPS1 protein. This variant has previously been reported to cause TRPS $1 .{ }^{1}$ TRPS 1 is a vertebrate transcription activator and repressor. ${ }^{1,3}$ The ability to repress or activate the target genes depends on the integrity of the Trps1 GATA-type zinc-finger domain and the C-terminal 119 amino acids of the protein, which contains two Ikaros-like zincfinger domains. ${ }^{4}$ Mutations in the GATA binding sites abolish TRPS1-mediated repression of target gene transcription, and the mutant proteins resulting from missense mutations have been shown to have a decreased affinity to DNA and function in a dominant negative manner. ${ }^{2}$ The mutation found in our patient is predicted to eliminate half of the DNA-binding domain and the rest of the TRPS1 protein, which has important functions. The other variant (c.2458C>G; p.Pro820Ala) found in the patient was also found in his unaffected father and sister, implying its non-pathogenicity.

Supernumerary teeth have been reported in patients with TRPS $1,{ }^{5-7}$ but only three patients were found to have TRPS1 mutations. $^{8,9}$ The supernumerary teeth found in our patient and the previously reported patients with TRPS1 mutations had the morphology of premolars. Because the incisors and molars were not formed when "functional" TRPS1 was absent in the patients, this suggests that TRPS1 is necessary for the formation of incisors and molars. Hypoplastic mandibular condyles with slender condylar necks were likely the results of TRPS1 mutation because Trps 1 is required for the development of the temporomandibular joints. ${ }^{10}$

Hypotrichosis in our patient appeared to be androgenic, similar to that of other patients with TRPS1. ${ }^{11}$ The first appearance of the axillary and pubic hair of our patient at puberty supports the influence of androgen. At 21 years of age, his scalp hair was fine, sparse and slow-growing, and more severely affected in the occipital area. During early hair morphogenesis, Trps1 regulates a number of transcription factors, Wnt inhibitors and extracellular matrix proteins., ${ }^{3,4}$ Patients with TRSP1 mutations or mice lacking Trps1 GATA domain have reduced expression of TRPS $1^{12}$ and Wnt inhibitors, activation of Wnt signaling, and subsequent fine hair and fewer hair follicles. ${ }^{4}$ In the mice lacking Trps1 GATA domain, increased Wnt signaling takes place only in the epithelial placode stage, but not in the later stages. ${ }^{3}$ The patients with TRPS1 have less hair because even though TRPS1 is downregulated, the Wnt signaling is not upregulated in the later stages of hair development to promote hair growth. In addition, there is also evidence showing that hair of the mice lacking GATA domain fail to be activated by a number of Wnt inhibitors in the epithelial placode at the initiation of hair follicle morphogenesis, leading to abnormal hair follicles as also seen in TRPS1 patients. ${ }^{3}$ Hair from the top of the head had a 
normal appearance. However, hair from the occiput had hypoplastic and twisted hair follicles with the rolling up of the scale edges of the hair cuticles. Hair in different parts of the scalp of the patients with TRPS1 have been shown to have different gene expression profiles. ${ }^{11}$

The rolling up of the scale edges of the hair cuticles has not been reported in patients with TRPS1. This is hypothesized to be due to the abnormal keratin composition of the hair cuticles, because keratins are the most abundant proteins of the hair cuticles and a number of genes related to keratins and hair morphogenesis including TRPS1 are downregulated in the balding scalp of patients with TRPS1. One of the genes that is downregulated in the hair of patients with TRSP1 is SOX21, a target gene of TRPS $1 .{ }^{13}$ Sox 21 is expressed in the cuticle layer and the progenitor cells of the hair shaft. This gene has a crucial role in hair morphogenesis by regulating the layered differentiation of hair follicles, and absence of Sox21 leads to hair loss. ${ }^{13}$ We hypothesize that the alopecia and the rolled up edges of hair cuticles in our patient are the effects of downregulation of SOX21 as a result of TRPS1 mutation because Sox21 has an important role in the interdigitation between hair cuticles and the inner root sheath. ${ }^{13}$

Congenital melanocytic nevus is known to be the result of postzygotic somatic mutations involving proteins in the mitogen-activated protein kinase pathway, especially NRAS and $B R A F$. It is believed that $C M N$ is a coincidence, and not related to TRPS1 mutation. The absence of body hair but increased number of hair cuticles of hair from CMN would suggest a link between increased melanin biosynthesis and prolonged anagen phase of hair follicles. It is noteworthy that increased melanogenesis in CMN overcomes TRPS1 mutation-associated hypotrichosis. This link is likely to involve a RET tyrosine kinase activity, because its role in extending the anagen phase of the hair cycle in association with upregulation of melanin production is known. ${ }^{14}$ In fact, this is supported by a study showing that transgenic mice with a constitutive activated RET have a prolonged anagen phase of hair follicles along with accelerated melanin production in and around hair bulbs. ${ }^{14}$ Upregulation of Shh and Edn1 in keratinocytes has been shown to cause hyperpigmentation and hypertrichosis. ${ }^{15}$ Therefore, $\mathrm{SHH}$ and $E D N 1$ could also play a role in the hypertrichosis reported herein. However, the rolling up of the scale edges of the hair cuticles, double mental foramina and abnormal condyles have not previously been reported.

ACKNOWLEDGMENTS: We thank the patients and their family for their kind cooperation and for allowing us to use their medical and dental information for research. This work was supported by The Thailand Research Fund; The Dental Association of Thailand; and The Faculty of Dentistry, Chiang Mai University.

CONFLICT OF INTEREST: None declared.

\section{REFERENCES}

1 Momeni P, Glöckner G, Schmidt O et al. Mutations in a new gene, encoding a zinc-finger protein, cause Trichorhinophalangeal syndrome type I. Nat Genet 2000; 24: 71-74.

2 Lüdecke HJ, Schaper J, Meinecke P et al. Genotypic and phenotypic spectrum in Trichorhinophalangeal syndrome types I and III. Am J Hum Genet 2001; 68: 81-91.

3 Fantauzzo KA, Christiano AM. Trps1 activates a network of secreted Wnt inhibitors and transcription factors crucial to vibrissa follicle morphogenesis. Development 2012; 139: 203-214.

4 Malik TH, Shoichet SA, Latham P, Kroll TG, Peters LL, Shivdasani RA. Transcriptional repression and developmental functions of the atypical vertebrate GATA protein TRPS1. EMBO J 2001; 20: 17151725.

5 Giedion A, Burdea M, Fruchter Z, Meloni T. Trosc V. Autosomaldominant transmission of the Trichorhinophalangeal syndrome. Report of 4 unrelated families, review of 60 cases. Helv Paediatr Acta 1973; 28: 249-259.

6 Kuna GB, Collipp PJ, Balsam D. Trichorhinophalangeal dysplasia (Giedion syndrome). A case report. Clin Pediatr (Phila) 1978; 17: 9698.

7 Niikawa N, Kamei T. The Sugio-Kajii syndrome, proposed Trichorhinophalangeal syndrome type III. Am J Med Genet 1986; 24: 759-760.

8 Kantaputra P, Miletich I, Lüdecke HJ et al. Trichorhinophalangeal syndrome with supernumerary teeth. J Dent Res 2008; 87: 1027-1031.

9 Kunotai W, Ananpornruedee P, Lubinsky M, Pruksametanan A, Kantaputra PN. Making extra teeth: Lessons from a TRPS1 mutation. Am J Med Genet Part A 2017; 173: 99-107.

10 Michikami I, Fukushi T, Honma S et al. Trps1 is necessary for normal temporomandibular joint development. Cell Tissue Res 2012; 348: 131-140.

11 Kim YJ, Yoon B, Han K, Park BC. Comprehensive Transcriptome Profiling of Balding and Non-Balding Scalps in Trichorhinophalangeal Syndrome Type I Patient. Ann Dermatol 2017; 29: 597601.

12 Ito T, Shimomura Y, Farooq M, Suzuki N, Sakabe J, Tokura Y. Trichorhinophalangeal syndrome with low expression of TRPS1 on epidermal and hair follicle epithelial cells. J Dermatol 2013; 40: 396-8.

13 Kiso M, Tanaka S, Saba R et al. The disruption of Sox21-mediated hair shaft cuticle differentiation causes cyclic alopecia in mice. Proc Natl Acad Sci USA 2009; 106: 9292-9297.

14 Kato M, Takeda K, Kawamoto $\mathrm{Y}$ et al. RET tyrosine kinase enhances hair growth in association with promotion of melanogenesis. Oncogene 2001; 20: 7536-7541.

15 Chitsazan A, Mukhopadhyay P, Ferguson B, Handoko HY, Walker GJ. Keratinocyte Cytokine Networks Associated with Human Melanocytic Nevus Development. J Invest Dermatol 2019; 139: 177-185.

\section{SUPPORTING INFORMATION}

Additional Supporting Information may be found in the online version of this article:

Figure S1. Electropherograms of a TRPS1 variant. A de novo heterozygous base substitution c.1842C $>\mathrm{T}$ is predicted to cause a stop codon at codon 615 and premature truncation of TRPS1 protein (p.Arg615Ter).

Figure S2. Electropherograms of a TRPS1 variant. A heterozygous base substitution (c. $2458 \mathrm{C}>\mathrm{G}$; p.Pro820Ala) is found in the patient and his unaffected father and sister. His mother has none of those variants. 